\title{
Dürers Zeichnung von der Beschießung des Hohenaspergs 1519 Der Künstler als Kriegsberichterstatter?"
}

\author{
Von Hermann Ehmer
}

Das Kupferstichkabinett der Staatlichen Museen zu Berlin verwahrt seit 1877 eine Federzeichnung von Albrecht Dürer ${ }^{1}$, die die Beschießung des Hohenaspergs 1519 darstellt. Die Zeichnung ist mit dem Monogramm des Künstlers, der Jahreszahl 1519 und eigenhändigen Beischreibungen als sein Werk ausgewiesen. Die Darstellung ist von der württembergischen Landesgeschichte ${ }^{2}$ schon seit längerer Zeit als „älteste Vedute, die ihre Entstehung einem bestimmten historischen Ereignis verdankt“" , wahrgenommen worden. Sie wurde zuletzt 2014 in der Ausstellung zum 500-Jahr-Gedenken des Tübinger Vertrags von 1514 in der Tübinger Kunsthalle gezeigt ${ }^{4}$. Auch in anderen Ausstellungen, die sich mit dem Thema Burg

* Die Arbeit geht zurück auf einen Vortrag beim Förderverein Hohenasperg in Asperg am 9. März 2015. Die dort vorgetragenen Ergebnisse wurden für die Publikation durch eingehende Forschungen wesentlich erweitert und mit Nachweisungen versehen.

${ }^{1}$ Inv. Nr. KdZ31. Die mit dunkelbrauner Tinte ausgeführte Federzeichnung misst 31,2 $\times$ $43,6 \mathrm{~cm}$. Zur Beschreibung vgl. Albrecht Dürer. Kritischer Katalog der Zeichnungen (Staatliche Museen Preußischer Kulturbesitz. Die Zeichnungen Alter Meister im Berliner Kupferstichkabinett), bearb. von Fedja Anzelewsky/Hans Mielke, Berlin 1984, S. 95, mit Abb. 92.

2 Schon Christoph Friedrich von STÄLIN, Württembergische Geschichte, 4. Teil, Stuttgart 1873, S.180, Anm.2, kannte diese Zeichnung, die mittels der damals neu erfundenen Photolithographie vervielfältigt worden war. Nach dem in der Königlichen Kupferstichsammlung (heute Staatsgalerie) befindlichen Exemplar wurde die Zeichnung abgebildet von Eugen Schneider (Hg.), Bilderatlas zur Württembergischen Geschichte, Esslingen 1913, S.77. Weitere Wiedergaben finden sich z. B. in: Ernst MülLER, Kleine Geschichte Württembergs, Stuttgart 1949, vor S. 81; Max Schefold, Alte Ansichten aus Württemberg, Stuttgart 1957, Bd.1, S. 20, Abb. Nr. 9; Bd. 2: Katalogteil, Nr. 222; Hans-Martin Maurer/Paul Sauer/ Werner FleischHauer u.a., Geschichte Württembergs in Bildern 1083-1918, Stuttgart/ Berlin/Köln 1992, S.107.

3 So Schefold (wie Anm. 2) Bd. 1, S. 20.

${ }^{4}$ Götz Adriani/Andreas Schmauder, 1514 Macht Gewalt Freiheit. Der Vertrag zu Tübingen in Zeiten des Umbruchs, Tübingen 2014, Nr.133, S.319, mit Abb. S.320f. Es handelt sich hier um die bislang beste Abbildung der Zeichnung, die nur wenig kleiner ist als das Original. 
beschäftigten, und den dazugehörigen Veröffentlichungen wurde die Dürersche Zeichnung vorgestellt und entsprechend gewürdigt ${ }^{5}$. Seit Hermann Römer (18801959), Professor am Lehrerinnenseminar in Markgröningen, 1929 die Herstellung einer Reproduktion in Originalgröße veranlasste und eine Begleitpublikation ${ }^{6}$ dazu veröffentlichte, hat auch die Geschichte von Asperg ${ }^{7}$ und der Orte der Umgebung, beginnend mit der Geschichte Markgröningens von Hermann Römer, sich mit der Zeichnung befasst und sie in den entsprechenden Publikationen abgebildet ${ }^{8}$. Im Gegensatz zur Landes- und Ortsgeschichte, für die die Zeichnung eine historische Quelle darstellte, hat sich die Dürerforschung noch wenig mit der Ansicht des Aspergs beschäftigt ${ }^{9}$. Der Grund dürfte darin liegen, dass dieses Blatt „in dieser Art einmalig im Werk Dürers ist ${ }^{\text {“10 }}$ und somit keine Vergleichsmöglichkeit bietet.

Auch ohne Dürers Bezeichnung „Asperg“, oben in der Mitte des Blattes, ist der Berg in der Ansicht von Süden deutlich zu erkennen. Auf der Zeichnung ist die Beschießung der Festung aus zahlreichen Rohren in vollem Gange: das Mündungs-

${ }^{5}$ So in der Ausstellung „Burg und Herrschaft“ des Deutschen Historischen Museums Berlin mit dem Katalog gleichen Titels, hg. von Rainer Atzbach/Sven LüKen/Hans ОттоMEYER, Berlin/Dresden 2010, wo die Dürer-Zeichnung auf S. 143 abgebildet ist, dazu der Kommentar auf S. 145. - Zu nennen ist ferner die Ausstellung „Mythos Burg“ des Germanischen Nationalmuseums Nürnberg. Katalog hg. von G. Ulrich GrossmanN, Nürnberg/ Dresden 2010. Wiedergabe der Zeichnung als Nr. 6.32, S. 262.

${ }^{6}$ [Hermann] Römer, Dürers Federzeichnung Asperg 1519 und die Beschießung des Hohenasperg durch die Schwäbischen Bundestruppen im Mai 1519, Ludwigsburg 1929. Die Übermittlung einer Kopie dieser Veröffentlichung ist Frau Gertrud Bolay in Asperg zu verdanken.

7 Theodor Bolay, Der Hohenasperg, Vergangenheit und Gegenwart, Bietigheim 1972, S. 16-18, mit Abb. S. 17; DERs., Chronik der Stadt Asperg, Bietigheim-Bissingen 1978, S. 35, mit Abb. S.34; Paul Sauer, Der Hohenasperg. Fürstensitz - Höhenburg - Bollwerk der Landesverteidigung, Leinfelden-Echterdingen 2004, S.81-83, mit Abb. S.83. Zuletzt: Ein feste Burg ist unser Gott. 450 Jahre Michaelskirche Asperg 1557-2007 [Evang. Kirchengemeinde Asperg 2007], mit Abb. S.19, wo allerdings eine Nachzeichnung wiedergegeben ist, auf der die Landschaft am rechten Rand ergänzt ist.

${ }^{8}$ Hermann Römer, Markgröningen im Rahmen der Landesgeschichte I, Markgröningen 1933, S.233-236, mit Abb. S.237; DERs., Geschichte der Stadt Bietigheim an der Enz, Stuttgart 1956, S.83f.; Hermann BurkhardT, Eglosheim zu Beginn der Neuzeit, in: Eglosheim. Ein Ort im Wandel der Jahrhunderte, hg. von Dems., Ludwigsburg 1991, S.110156, hier S. 128-131, mit Abb. S. 129; Robert KreTzschmar, Möglingen im Mittelalter und zu Beginn der frühen Neuzeit, in: Albrecht GüHring, Möglingen. Pforte zum Strohgäu, Möglingen 2000, S.57-90, hier S. 82-84, mit Abb. S. 83.

9 Heinrich Wölfflin, Die Kunst Albrecht Dürers, München 1. Aufl. 1905, 6. Aufl. 1943, erwähnt die Zeichnung nicht. Ein Gleiches gilt für Friedrich WinkLER, Albrecht Dürer. Leben und Werk, Berlin 1957, wo die Belagerung von Hohenasperg zwar im Register erwähnt wird, die Seitenangabe aber in die Irre führt. Unerwähnt bleibt das Blatt auch bei Franz Winzinger, Albrecht Dürer in Selbstzeugnissen und Bilddokumenten (Rowohlts Monographien), Reinbek bei Hamburg 1971.

10 Albrecht Dürer. Kritischer Katalog (wie Anm.1) S. 95. 
feuer ist zu erkennen, ebenso die fliegenden Kanonenkugeln. Die Kanonade zeigt bereits beträchtliche Wirkungen, die Südseite der Umfassungsmauer der Festung weist eine riesige Bresche auf, zahlreiche weitere Einschüsse sind sichtbar, auch die südwestliche Ecke der Ummauerung scheint stark beschädigt.

Die Jahreszahl 1519 zeigt an, dass es sich um ein Ereignis des Feldzugs des Schwäbischen Bundes gegen Herzog Ulrich von Württemberg handelt, in dessen Verlauf die Festung beschossen wurde. Der heutige Betrachter der Zeichnung, der an Foto- und Filmaufnahmen tatsächlicher oder nachgestellter Kriegshandlungen gewöhnt ist, bekommt den Eindruck, dass sich Dürer irgendwo auf dem südlich vom Asperg ansteigenden Gelände, dem Siechenberg, der sich zum Kleinaspergle hinaufzieht, mit seinem Skizzenblatt niedergelassen hat, um - aus sicherer Entfernung - das kriegerische Geschehen mit seiner Zeichenfeder festzuhalten.

So weit scheint alles klar zu sein. Die Ortsgeschichte, die sich in erster Linie mit der von der Zeichnung gebotenen Topographie befasste, hat jedoch einige Fragen aufgeworfen, die bislang nicht in jedem Fall zufriedenstellend beantwortet werden konnten. Die Probleme liegen hier also im Detail. Die vorliegende Arbeit will sich diesen in einem Dreischritt nähern. Zunächst soll der Anlass dieser kriegerischen Auseinandersetzung in seinem geschichtlichen Zusammenhang dargestellt werden. Sodann wird der Frage nachgegangen, wie und warum Dürer - und ob er überhaupt - an den Ort des Geschehens gekommen ist. Erst dann soll auf die Einzelheiten seiner Zeichnung von der Beschießung des Asperg eingegangen werden.

\section{I.}

Der Anlass des Feldzugs des Schwäbischen Bundes in Württemberg war die Einnahme der Reichsstadt Reutlingen durch Herzog Ulrich nach kurzer Belagerung Ende Januar $1519^{11}$. Kurz zuvor, am 12. Januar, war Kaiser Maximilian gestorben, und der Herzog gedachte, die kaiserlose Zeit zu nutzen, um die Reichsstadt zu vereinnahmen. Schließlich gab es mehrere Anwärter für die Kaiserkrone, die sich vorerst gegenseitig in Schach halten würden. Ansonsten hatte Herzog Ulrich seinen Handstreich auf Reutlingen aber diplomatisch kaum abgesichert, so dass er davon überrascht wurde, dass der Schwäbische Bund sich tatkräftig für Reutlingen einsetzte. Der Bund hatte bereits auf einem Bundestag in Augsburg an Antonii [17. Januar] aufgrund des Todes des Kaisers Vorsichtsmaßnahmen wegen allerlei Empörung im Reich beschlossen, insbesondere sollte jeder Bundesstand eine Botschaft an die Eidgenossen senden, um diese zu bewegen, dem Herzog von Württemberg keine Hilfe zu leisten. Auch Stände außerhalb des Bundes sollten aufgefordert werden, dem unbilligen Fürnehmen des Herzogs gegen Reutlingen

${ }^{11}$ Zum Folgenden vgl. Ludwig Friedrich Heyd, Ulrich, Herzog zu Württemberg, Bd.1, Tübingen 1841, S. 523-566. 
entgegenzutreten ${ }^{12}$. Nach den bereits getroffenen Vorbereitungen hatte der Bund alsbald ein Heer gesammelt, das unter der Führung von Herzog Wilhelm von Bayern (1511-1550), dem Schwager Herzog Ulrichs, im April 1519 in Württemberg einmarschierte.

Über den Feldzug berichtet die Chronik des Friedrich Stumphart von Cannstatt, seit 1534 Vogt zu Böblingen ${ }^{13}$. Stumphart ${ }^{14}$ hat die Chronik unter dem Datum Böblingen, 15. September 1534, Herzog Christoph, dem Sohn Herzog Ulrichs, gewidmet. Das Werk ist in 15 verschieden große Artikel oder Abschnitte eingeteilt. Dies zeigt, dass hier unterschiedliches Material versammelt ist, darunter etliche Mandate und gedruckte Ausschreiben Herzog Ulrichs ${ }^{15}$, die öffentlich zugänglich waren. Es ist somit deutlich, dass Stumphart als Redaktor, nicht als Verfasser dieser Textsammlung zu bezeichnen ist.

Der dritte und längste Artikel der Stumphartschen Arbeit handelt von dem Feldzug des Schwäbischen Bundes gegen Herzog Ulrich im Frühjahr 1519, wobei die Ereignisse in der Regel Tag für Tag angegeben werden ${ }^{16}$. Für diese Beschreibung des Feldzugs hat Stumphart auf den Bericht eines Feldzugsteilnehmers auf der bündischen Seite zurückgegriffen, der noch 1519 in Augsburg gedruckt worden ist ${ }^{17}$. Diesen Bericht hat Stumphart als guter Württemberger an einigen Stellen

12 Karl KLÜPfel, Urkunden zur Geschichte des Schwäbischen Bundes (1488-1533), Teil 2, Stuttgart 1853, S. 158 f.

13 Abdruck bei Christian Friedrich SatTlen, Geschichte des Herzogthums Würtenberg unter der Regierung der Herzogen, 2. Teil, Ulm 1770, Beilage 21, S.30-62 nach Stumpharts Autograph in HStA Stuttgart J 1 Bd. 36, Bll.370 r-432 r.

${ }_{14} \mathrm{Zu}$ ihm vgl. Gustav Bossert [d.J.], Friedrich Stumphart, der Verfasser einer Chronik über Herzog Ulrichs Verjagung, in: ZWLG 5 (1941) S.244-254. Bossert bietet zunächst einen Überblick über die 15 Artikel des Werks, sodann Daten zur Biographie Stumpharts und befasst sich dann mit dem 9. Artikel, einem Gespräch über Herzog Ulrich, und dem 11. Artikel, der über Misshandlungen der Anhänger Herzog Ulrichs 1524 handelt. Zum Schluss druckt er noch einige Quellen aus den Jahren 1539/1540 über die Verleihung des Mesnerdienstes in Meimsheim an Stumphart ab. - Nach Walther Pfeilsticker, Neues Württembergisches Dienerbuch, Bd.2, Stuttgart 1963, \2232 war Friedrich Stumphart seit Montag nach Lucie [14. Dezember] 1534 Vogt in Böblingen. Bereits am 11. Mai 1536 wird er als „alter Vogt“ genannt. 1537/1538 war Stumphart Amts- und Stadtschreiber in Lauffen am Neckar; Pfeilsticker, ebd., $\$ 2525$.

15 Sattler hat diese Stücke, da er sie schon anderweitig verwendet hatte, in seiner Edition der Stumphartschen Chronik weggelassen.

${ }^{16}$ Römer, Dürers Federzeichnung (wie Anm. 6) S. 4 und 8 spricht daher von einem Feldoder Kriegstagebuch.

17 Des hochlobliche[n] | schwebischen pu[n]ds | Hörzug im landt zů Wirtenberg | mit ordenlichen tagraysung $\mid$ vn[d] etlichen geschichte[n] wie $\mid$ sich Stät[,] flecke[n] vn[d] vogt | tey täglich ergeben ha | ben kürtzlich begrif $\mid$ fen Vnd lustig | zůlesen | M D XIX Jar. Ohne Drucker: [Augsburg] 1519. $4^{\circ}, 20$ ungezählte Seiten, Kustoden: [A1-4], B [1-4], C [1-2]. VD16 H 3988. - Sattler kannte diesen Druck offensichtlich nicht. Für ihn stellte Stumpharts Bericht die authentische Quelle dar. 
verändert; so geht schon im ersten Satz der gut fromm bertzog auf seine Rechnung. Der unbekannte Verfasser des Berichts, der wohl in der unmittelbaren Umgebung Herzog Wilhelms von Bayern zu suchen ist, redet an dieser Stelle von der $A b-$ sagung an Herzog Ulrich, also der Kriegserklärung des Bundes. Stumphart hat vor allem die Ortsnamen, die der des Landes unkundige Autor einigermaßen verballhornt angegeben hat, bereinigt. Aber auch sonst hat er in den ihm vorliegenden Text eingegriffen, insbesondere hat er, um seine württembergische Tendenz zu bekräftigen, einiges hinzugefügt, anderes weggelassen. Zu Stumpharts Zutaten gehört die Episode vom Marsch des Bundesheers von Weißenstein ins Filstal hinunter, der unordentlich vor sich gegangen sei, weil man meinte, das Land schon in der Tasche zu haben. Hierher gehört auch die Geschichte von dem silbernen Dolch, den Herzog Wilhelm im Lager im Neckartal verloren hatte und nicht wieder bekam. Stumphart hat eigenartigerweise die Tagesdaten, die der Druck getreulich bietet, weggelassen, aber immerhin die Wochentage benannt.

Es soll hier der redaktionellen Arbeit Stumpharts nicht weiter nachgegangen werden. Nach Bossert war Stumphart 1514 bis 1519 Vogt in Maulbronn ${ }^{18}$. Dies wird deutlich in dem Bericht über den 11. April, als das Bündische Heer im Neckartal lagerte. Hier wurden an die Städte und Dörfer, die sich ergeben hatten, gedruckte und unterschriebene Wappenschilde mit den bayerischen Rauten und dem roten Tatzenkreuz des Schwäbischen Bundes ${ }^{19}$ ausgegeben, damit sie nachmalß sicher sein möchten, umb dise Wappen hatt jederman gelt geben müssen, on die von Mulbron denen hatt mans geschenckt byß an xii guldin. Der hier wiedergegebene Abdruck des Stumphartschen Textes bei Sattler ${ }^{20}$ ist, auch wegen der fehlenden Interpunktion, missverständlich. Der Satz macht nur dann Sinn, wenn man on die von Mulbron, denen hatt mans geschenckt als Einschub versteht, der auf Stumphart selber zurückgeht. In dem 1519 in Augsburg gedruckten Text ist natürlich von den Gebühren für die Salvaguardia oder beschütz wappen keine Rede.

Nach dem von Stumphart wiedergegebenen Bericht versammelte sich das Bündische Heer am Sonntag Oculi [27. März] in Langenau bei Ulm, rückte zunächst vor Heidenheim, wo sich das Schloss am 29. März nach einer Beschießung ergab. Über Weißenstein zog man hierauf die Steige hinunter ins Filstal bis Süßen und rückte am 1. April vor Göppingen, das sich nach einem Schusswechsel ergab. Am 3. April ergab sich auch das Schloss Teck. Vor Kirchheim wurde das Bündische Heer abgewiesen, weshalb es weiterzog und sich bei Denkendorf lagerte. Am 6. April schlug man ein Lager im Neckartal bei Untertürkheim auf. In den folgenden Tagen ergaben sich Stuttgart und Cannstatt dem Bund, ebenso weitere Städte

\footnotetext{
18 Bossert (wie Anm. 14) S. $245 \mathrm{f}$.

${ }^{19}$ Herzog Wilhelm hatte laut seiner Bestellung vom 5. April in Esslingen 400 solcher Wappenbriefe verfertigen lassen; KLÜPFEL (wie Anm.12) S.167.

20 Sattler (wie Anm.13) S. 35.
} 
und Dörfer des Landes. Schorndorf ergab sich am 10. April nach einer Beschießung. In der folgenden Woche zog das Heer über Möhringen und Vaihingen nach Ehningen bei Böblingen und lagerte sich am 14. April vor Tübingen. Die Stadt ergab sich sogleich, mit der Besatzung des Schlosses wurde nach einem Schusswechsel ein Waffenstillstand zwischen Karfreitag [22. April] und Ostermontag [25. April] vereinbart, nach dessen Beendigung sich die Besatzung des Schlosses ergab $^{21}$.

Das Heer zog in den folgenden Tagen ins württembergische Unterland bis Möckmühl, Weinsberg und Neuenstadt, um sodann vor den Asperg zu rücken. Von einer Gegenwehr Herzog Ulrichs verlautet im Übrigen nichts, lediglich die festen Plätze machten Anstalten zur Verteidigung, ergaben sich aber meist nach kurzer Zeit. Die Belagerung des Tübinger Schlosses dauerte wegen der Waffenruhe zwischen Karfreitag und Ostermontag länger, aber auch deswegen, weil sich im Schloss die beiden Kinder Herzog Ulrichs, Anna und Christoph, befanden. Dies ist der Grund, weshalb der Herzog davon überzeugt war, dass es zu früh aufgegeben wurde ${ }^{22}$. Länger dauerte aber auch die Belagerung des Asperg, weshalb diese in der Chronik der Ereignisse den weitaus breitesten Raum einnimmt.

\section{II.}

Zur Beantwortung der Frage, wie und warum Albrecht Dürer 1519 zum Asperg kam, ist vorauszuschicken, dass die Nachrichten über Dürer aus der Zeit zwischen dem Augsburger Reichstag 1518 und der Niederländischen Reise 1520 dünn gesät sind. Einer seiner Biographen bezeichnet deshalb diese Jahre als „Zeit der Besinnung" 23 .

Ein Blatt, auf dem sich Dürer als Kranker darstellte, der auf eine schmerzende Stelle seines Oberbauchs deutet, ist auf 1519 datiert. Deutet Dürer auf seine linke Seite, müsste es sich um die Milz handeln, was aber wenig wahrscheinlich ist. Handelt es sich aber - was näher liegt - um ein Spiegelbild, muss es die Galle sein, die ihm Beschwerden verursachte ${ }^{24}$. Doch lässt sich dieses Selbstbildnis des kranken Dürer nicht näher datieren, so dass es für die Reise, die ihn zum Asperg führte, keinen weiterführenden Hinweis bietet.

21 Adriani/Schmauder (wie Anm. 4) S. 404.

${ }^{22}$ Vgl. die "Schandtafel“ mit den Namen der 64 Adligen, die die Schlossbesatzung bildeten, die Herzog Ulrich nach seiner Rückkehr 1534 im Rittersaal des Schlosses anbringen ließ; Adriani/Schmauder (wie Anm.4) S.404f.

${ }^{23}$ Fedja Anzelewsky, Dürer. Werk und Wirkung, Stuttgart 1980, S. 195.

${ }^{24}$ Für den Hinweis, dass es sich um ein Spiegelbild handeln muss, und für medizinische Beratung in diesem Fall ist Herrn Dr. med. Ingo Lenk in Fellbach-Schmiden zu danken. 
Im Frühjahr 1519 scheint im Briefwechsel zwischen Willibald Pirckheimer und Lorenz Beheim der Plan Dürers auf, Spanien und England zu besuchen ${ }^{25}$. Die Freunde scheinen ihm mit Hinweis auf seine zarte Konstitution und die Gefahren der Reise davon abgeraten zu haben. Diese Reisepläne fanden daher keine Verwirklichung. Der Zweck einer solchen Reise sollte für Dürer zweifellos sein, neue Anregungen zu sammeln und sich der Welt bekannt zu machen, um so neue Verdienstmöglichkeiten zu finden, nachdem Kaiser Maximilian, sein langjähriger Mäzen, verstorben war. Es ist daher leicht einzusehen, dass Dürer, um seine Reiselust zu befriedigen, sich einer Gesandtschaft nach Zürich, bestehend aus seinem Freund Willibald Pirckheimer und Martin Tucher, angeschlossen hat und so nach Württemberg gekommen ist ${ }^{26}$.

Um den genauen Zeitpunkt der Reise festzustellen, sind die verfügbaren Quellen zu befragen. Hans Rupprich, der den „Nachlass“ Dürers herausgegeben hat, nämlich nicht nur seine autobiographischen Schriften und seinen Briefwechsel, sondern überhaupt alle greifbaren Zeugnisse zu seinem persönlichen Leben ${ }^{27}$, zeigt, dass Dürer am 12. Mai 1519 als Zeuge bei der Beurkundung eines Hausverkaufs $^{28}$ in seiner Nachbarschaft auftrat, folglich an diesem Tag noch in Nürnberg gewesen sein muss. Lorenz Beheim gratulierte am 7. Juli 1519 aus Bamberg seinem Freund Willibald Pirckheimer und Dürer zur glücklichen Heimkehr aus der Schweiz ${ }^{29}$. Da die Kunde von der Rückkehr der Nürnberger erst nach Bamberg gelangen musste, eröffnen die beiden Daten somit ein Zeitfenster von rund sechs Wochen für die Reise, nämlich von Mitte Mai bis Ende Juni.

Es sollte aber möglich sein, diesen Zeitraum noch näher einzugrenzen. Da es sich um eine offizielle Gesandtschaft der Reichsstadt Nürnberg nach Zürich handelte, müsste es an beiden Orten noch Korrespondenzen oder andere Unterlagen über diese Angelegenheit geben. Am naheliegendsten wäre natürlich eine Instruktion

${ }^{25}$ Dürer. Schriftlicher Nachlass, Bd. 1: Autobiographische Schriften/Briefwechsel/Dichtungen. Beischriften, Notizen und Gutachten. Zeugnisse zum persönlichen Leben, hg. von Hans Rupprich, Berlin 1956, B Nr. 50, S. 263: Lorenz Beheim an W. Pirckheimer, [Bamberg] 23. März 1519. Vgl. jetzt die Edition in: Willibald Pirckheimers Briefwechsel, Bd.4, bearb. und hg. von Helga Scheible, München 1997, Nr.597, S.39-41, hier S.40: Quod Albertonus intendit proficisci in Angliam vel miseram Hyspaniam, credo quod fata ipsum agant. Non est ipse iam de invenibus. Est delicatus factus, non poterit sufferre incommoda tanti itineris. Taceo qualitatem alieni aeris. Sic sapit, serviat uxori suae, e contra ipsa sibi. Cum non habeat filios, sufficiat sibi sua substantia et quietos iam agat dies Deo serviendo.

26 Anzelewsky (wie Anm.23) S. 206.

27 Dürer. Schriftlicher Nachlass (wie Anm. 25).

28 Dürer. Schriftlicher Nachlass (wie Anm. 25) S. 248.

29 Dürer. Schriftlicher Nachlass (wie Anm. 25) S. 264: Lorenz Beheim an W. Pirckheimer, [Bamberg] 7. Juli 1519, desgleichen in Willibald Pirckheimers Briefwechsel, Bd.4, Nr.608, S.59-61, hier S.59: Salvum te redisse cum tuo Alberto et gandeo et gratulor, rebus, ut intellego, bene gestis et expeditis. ... Placet, quod Helvetii vos egregie tractarunt et bene quidem, quia baec concordia proderit eis non parum, ni fallor. 
der Gesandten oder eine Reisekostenabrechnung, aus der womöglich sogar der Reiseweg zu entnehmen wäre. Doch ist die Nürnberger Stadtrechnung von 1519 nicht mehr erhalten, und auch in den Stadtrechnungsbelegen findet sich kein entsprechender Hinweis ${ }^{30}$. Doch ist es möglich, noch anderweitige Spuren dieser Angelegenheit zu finden.

Am 13. Mai 1519 fragte Nürnberg in Zürich an, wann und wo die nächste Zusammenkunft der Sendboten der Eidgenossenschaft sein würde, da man entschlossen sei, dazu eine Gesandtschaft abzufertigen ${ }^{31}$. Die Antwort aus Zürich traf am 27. Mai 1519 oder kurz zuvor in Nürnberg ein. Bürgermeister und Rat zu Nürnberg dankten nämlich mit Schreiben vom 27. Mai ihren Züricher Kollegen für die Auskunft und kündigten eine Gesandtschaft an, die am 5. oder 6. Juni in Zürich eintreffen sollte ${ }^{32}$. In diesem Beglaubigungsschreiben, dessen Ausfertigung in Zürich erhalten ist ${ }^{33}$, werden freilich die Gesandten nicht namentlich genannt. Immerhin bat Nürnberg darum, ihre Gesandtschaft uff 12 pferd ungeverlich mit zymlich herberg zu versehen.

Es fragt sich natürlich, welchen Zweck diese stattliche Gesandtschaft verfolgte ${ }^{34}$. Schließlich war man in einer Zeit politischer Hochspannung. Die Kaiserwahl war in der Schwebe. Es war bekannt, dass der französische König nach der Kaiserkrone strebte und womöglich die Eidgenossen auf seine Seite ziehen wollte. Vor allem aber meldete der spanische König, der nachmalige Kaiser Karl V., seine Ansprüche an. Zum anderen war gerade der Feldzug des Schwäbischen Bundes gegen Herzog Ulrich von Württemberg im Gange und auch in dieser Angelegenheit wurden die Schweizer von beiden Seiten umworben.

Die Tagung der Sendboten der Eidgenossen, zu der die Nürnberger Gesandtschaft abgefertigt worden war, fand am 3. Juni 1519 in Zürich statt. In dem darüber ausgefertigten Abschied ${ }^{35}$ wird das Anliegen der Nürnberger genannt. Es war nun

30 Freundliche Auskunft des Staatsarchivs Nürnberg vom 25.3.2015. - Herrn Leitender Archivdirektor Prof. Dr. Peter Fleischmann ist für freundliche Hilfestellung in dieser Angelegenheit zu danken.

31 StA Nürnberg, Reichsstadt Nürnberg, BB 80, Bl.22 r-v, Konzept.

32 StA Nürnberg, Reichsstadt Nürnberg, BB 80, B1.37 r-v, Konzept.

${ }^{33}$ StA Zürich A 202.5. - Herrn Lic. phil. Martin Leonhard vom Staatsarchiv des Kantons Zürich habe ich herzlich für seine Recherche und die weiterführenden Hinweise zu danken.

${ }^{34}$ Einen Hinweis gibt die Autobiographie Pirckheimers, vgl. Willibald Pirckheimers Schweizerkrieg. Nach Pirckheimers Autographum im Britischen Museum hg. von Karl RÜcK, München 1895, S. 148: Per aliquos igitur annos senatorio munere quiete est perfunctus nec ullis postea degranatus est legationibus, praeterquam semel ad Eluetios missus, cum bellum inter marchionem et Nurenbergenses rursus glisceret. Qua etiam legatione et subsidii petitione marchio deterritus a proposito destitit et rursus cum Nurenbergensibus in gratiam rediit.

${ }^{35}$ Die Eidgenössischen Abschiede aus dem Zeitraume von 1500 bis 1520, bearb. von Anton Philipp SEgesser (Amtliche Sammlung der ältern Eidgenössischen Abschiede, Bd.3,2), Luzern 1869, Nr.780, S.1166-1170, hier S.1168, lit. n. 
keines der beiden genannten reichspolitischen Probleme, sondern eines, das die Nürnberger unmittelbarer betraf. Markgraf Kasimir von Brandenburg (1481 -1527) hatte bei Nürnberg einen neuen Zoll eingerichtet, ohne Rücksicht auf Freiheiten und Herkommen der Nürnberger und ohne auf ihr Angebot einer rechtlichen Auseinandersetzung deswegen vor den Kurfürsten oder vor den Eidgenossen einzugehen. Die Stadt müsse daher befürchten, so heißt es in dem Eidgenössischen Abschied, gänzlich unterdrückt zu werden, was aber auch anderen Städten geschehen könne.

Es handelte sich also bei dem Gegenstand der Nürnberger Gesandtschaft nach Zürich um ein Kapitel in der ständigen Auseinandersetzung zwischen der Reichsstadt und der Markgrafschaft, in dem man sich des Beistands auch der Eidgenossen versichern wollte ${ }^{36}$. Die Nürnberger Gesandtschaft gelangte zwar nicht mehr rechtzeitig zu der Tagsatzung nach Zürich, doch wurde ihr Anliegen in den Abschied aufgenommen, um es bei der künftigen Tagsatzung zu Baden (im Aargau) zu verhandeln. Der Tag in Baden fand vier Wochen später, am 5. Juli 1519 statt. Hinsichtlich des Nürnberger Ansuchens wurde dort beschlossen, dem Markgrafen Kasimir zu schreiben, dass er die Nürnberger nicht mit seinem neuen Zoll beunruhigen, sondern sie bei ihrem alten Herkommen und ihrem Rechterbieten lassen möge ${ }^{37}$.

Die Nürnberger Gesandten hatten in Zürich erklärt, dass sie nicht auf die Badener Tagsatzung warten könnten, weshalb man in Aussicht stellte, ihnen die Antwort schriftlich nachzusenden. Jedenfalls bedankte sich die Reichsstadt Nürnberg am 24. Juni 1519 bei den Zürichern in aller Form für die freundliche Aufnahme ihrer Gesandten ${ }^{38}$. Demnach waren diese an jenem Tag oder kurz zuvor wieder in Nürnberg eingetroffen.

Wir haben hier auf die Nürnberger Sorgen wegen des markgräflichen Zolls nicht weiter einzugehen, zumal der Markgraf aufgrund der diplomatischen Aktivitäten der Nürnberger von seinem Vorhaben abstand ${ }^{39}$. Es geht hier lediglich um den mutmaßlichen Zeitpunkt, an dem Dürer am Asperg gewesen sein kann. Immerhin hat sich das Zeitfenster durch die vorstehende Untersuchung weiter eingeschränkt. Klar dürfte auch sein, dass die Gesandten wegen des Zeitdrucks, da man ohnehin zu spät dran war, den Hinweg nicht über Württemberg genommen haben können.

${ }^{36}$ Die Sache war bereits Gegenstand von Verhandlungen auf dem Bundestag in Augsburg im Januar gewesen, wie der Abschied vom 17. Januar 1519 dartut; Klüpfel (wie Anm. 12) S. 159 .

37 Die Eidgenössischen Abschiede aus dem Zeitraume von 1500 bis 1520 (wie Anm.35), Nr. 783, S. 1172-1177, hier S. 1172 lit. d.

38 StA Zürich A 202.5, Ausfertigung.

39 Wie aus einem Schreiben der Nürnberger an Herzog Wilhelm von Bayern vom 13. Mai 1519 hervorgeht, Konzept, StA Nürnberg, Reichsstadt Nürnberg, BB 80, Bl.22v-23 r, sollte die Nürnberger Botschaft auf dem Bundestag zu Esslingen, der wegen der Württemberger Angelegenheit einberufen worden war, diese Sache dort ebenfalls vorbringen. 
Der wahrscheinlichste, weil kürzeste Reiseweg von Nürnberg nach Zürich führte zweifellos über Ulm oder Augsburg. Im Übrigen waren die kriegerischen Verwicklungen in Württemberg, wie gewiss auch in Nürnberg bekannt war, inzwischen beendet.

Jedenfalls hat Albrecht Dürer die ganze Reise nach Zürich mitgemacht, obwohl seine Teilnahme nur mittelbar belegt werden kann. Deutlich wird dies durch einen Brief Pirckheimers an Ulrich Zwingli vom 23. November 1519, in dem er dem nachmaligen Züricher Reformator Grüße von Dürer bestellt, der jetzt nicht nur Pirckheimers, sondern auch Zwinglis Freund ist ${ }^{40}$. Überhaupt scheinen die Nürnberger die Gelegenheit genutzt zu haben, sich mit der gelehrten Welt Zürichs bekannt zu machen. Ein weiteres Zeugnis dafür ist Dürers Brief vom 6. Dezember 1523 an Felix Frey, Propst des Großmünsterstifts in Zürich ${ }^{41}$, dem er für ein ihm übersandtes Büchlein dankt und ihm die gewünschte Zeichnung eines „Affentanzes“ zuschickt. Dürer bittet Frey, Grüße an Ulrich Zwingli, Hans Leu, Hans Urich und die anderen Bekannten zu bestellen. Die Freunde mögen die mitfolgenden fünf Stücke Kunstblätter untereinander teilen.

Nur auf der Rückreise von Zürich konnten die Nürnberger, da nun keine Eile geboten war und die Antwort der Eidgenossen ohnehin schriftlich nachfolgen würde, einen Abstecher über Württemberg machen. Wenn die Nürnberger Gesandten also am 5./6. Juni in Zürich eintrafen und - wie die geschlossenen Bekanntschaften zeigen - auch mehrere Tage dort verweilten und am 24. Juni wieder daheim in Nürnberg waren, konnten sie nur Mitte Juni 1519 in Württemberg gewesen sein. Möglicherweise war es ein besonderer Auftrag, der die Nürnberger Gesandtschaft nach Württemberg geführt hat. $\mathrm{Zu}$ denken wäre hier an einen Besuch des Bundestags in Esslingen, von dem jedoch ein Abschied vom 18. Mai überliefert ist, wonach man sich auf den 12. Juni nach Nördlingen vertagte, aber offensichtlich noch länger in Esslingen beieinander blieb ${ }^{42}$.

40 Willibald Pirckheimers Briefwechsel, Bd.4, Nr.636, S.127-129, hier S.128: Albertus non iam meus sed et tuus te salutat.

${ }^{41}$ Dürer. Schriftlicher Nachlass (wie Anm. 25) Nr.51, S. 106-108.

42 Einen Beleg dafür bietet die Feldzugschronik, wonach Georg von Frundsberg am 24. Mai nach Esslingen ritt, um sich mit Herzog Wilhelm von Bayern und den anderen Bündischen, die dort versammelt waren, wegen der Bedingungen der Übergabe des Aspergs zu unterreden; Hörzug (wie Anm.17), Bl.C1r; SATTler (wie Anm.13) S.42. - Hierher gehört möglicherweise auch der von Klüpfel (wie Anm.12) S.162f. unzweifelhaft falsch eingereihte Abschied der Bundesversammlung. Leider hilft Horst CARL, Der Schwäbische Bund 1488-1534. Landfrieden und Genossenschaft im Übergang vom Spätmittelalter und Reformation (Schriften zur südwestdeutschen Landeskunde, Bd.24), LeinfeldenEchterdingen 2000, der die Unzuverlässigkeit des Klüpfelschen Werks S.6-9 beklagt, aufgrund seines strukturgeschichtlichen Ansatzes hier nicht weiter. 
Der Zeitraum, innerhalb dessen Albrecht Dürer am Asperg gewesen sein kann, ist somit auf rund eine Woche Mitte Juni 1519 eingeschränkt ${ }^{43}$. Jedenfalls kann er erst zu einem Zeitpunkt dort gewesen sein, an dem die Beschießung des Asperg bereits zwei oder drei Wochen zurücklag. Gewiss waren aber die Artilleriestellungen und die durch die Beschießung bewirkten Schäden noch zu sehen, und sicher fand sich jemand, der Interessierten das Geschehen schildern konnte. Auf jeden Fall haben wir mit Dürers Zeichnung keine Schilderung eines Augenzeugen, die mit einer fotografischen Aufnahme vergleichbar wäre, auch wenn hier feuernde Geschütze und fliegende Kanonenkugeln zu sehen sind. Gleichwohl wird nach dem Zweck dieser Darstellung zu fragen sein.

\section{III.}

Es ist bekannt, dass die Landschaft als solche nur zögerlich in die Kunst Eingang gefunden hat. Von Dürer kennen wir einige wenige Landschaften und Ansichten aus Nürnberg und Umgebung, dann von seinen Reisen, so nach Italien, wo er besonders Alpenlandschaften im Bild festgehalten hat ${ }^{44}$. Am fruchtbarsten war die Niederländische Reise, doch mag dies in erster Linie mit der Überlieferungslage zusammenhängen. In den Niederlanden scheint Dürer auch die Manier kennengelernt zu haben, Porträts mit Landschaften oder Städten als Hintergrund zu versehen. Sodann bilden Landschaften traditionell - und so auch bei Dürer - hauptsächlich den Hintergrund für andere Darstellungen, etwa der biblischen Geschichte und für Heiligenbilder. Viele von Dürers Werken zeigen im Hintergrund Burgen, Bergstädte und dergleichen ${ }^{45}$. Man darf daher schon ein allgemeines Interesse Dürers am Asperg als Bergfestung annehmen.

Am Beispiel des Aquarells der oberitalienischen Bergstadt Arco (nördlich des Gardasees) hat Hermann Leber ${ }^{46}$ den Zusammenhang dieser Darstellung mit Dürers Proportionenlehre aufgezeigt und an diesem Fall dargelegt, dass es sich hier um ein in der Werkstatt und nicht um ein vor dem Gegenstand entstandenes Werk handelt. Ob dieser Zusammenhang mit der Proportionenlehre auch der Zeichnung des Aspergs zugrunde liegt, kann hier nicht untersucht werden. Es soll hier zu-

${ }^{43}$ Die Reise ist somit in Albrecht Dürer. Kritischer Katalog (wie Anm.1) S. 95, vier Wochen zu früh angesetzt, ebenso bei Anzelewsky (wie Anm. 23) S. 205, Abb. 194.

${ }_{44}$ Dazu jetzt Daniel Hess, Die Natur als vollkommene Lehrmeisterin der Kunst, in: Der frühe Dürer, hg. von Daniel Hess/Thomas Eser, Nürnberg 2012, S. 117-131, besonders S.120-125; G. Ulrich Grossmann, Die Architektur im Werk des jungen Dürer, in: ebd., S. $221-235$.

${ }^{45}$ Grossmann, Die Architektur (wie Anm. 44) S. $232 \mathrm{f}$.

${ }^{46}$ Hermann Leber, Albrecht Dürers Landschaftsaquarelle. Topographie und Genese, Hildesheim/Zürich/New York 1988; Grossmann, Die Architektur (wie Anm. 44) S. 226. 
nächst darum gehen, die topographischen Probleme, die diese Zeichnung aufwirft, zu beleuchten.

Bei den Landschaftsaquarellen Dürers aus Franken, die teilweise schon früh identifiziert wurden, konnte, insbesondere beim „Tal bei Kalchreuth“, gezeigt werden ${ }^{47}$, dass es sich hier nicht um eine Landschaftsdarstellung handelt, die mit einem Foto vergleichbar wäre. Vielmehr hat hier eine wohldurchdachte künstlerische Verarbeitung des Landschaftseindrucks stattgefunden. Insbesondere ist eine Steigerung der Höhen und eine Verkürzung der Breitenausdehnung der Landschaft festzustellen. Diese Darstellungsweise bedingt verschiedene Standorte des Künstlers, der dadurch den räumlichen Eindruck seiner Arbeit steigerte. Diese Vereinigung der Ansichten aus verschiedenen Standorten zu einem stimmigen Gesamtbild wird besonders deutlich in dem Kupferstich „Nemesis“ oder „Das große Glück“48, in dem die die Landschaftsdarstellung zu Füßen der Frauenfigur die Stadt Klausen im Etschtal zum Vorbild hat ${ }^{49}$. Gerade hier ließ sich zeigen, dass die gebotene Ansicht nicht von einem einzigen Standpunkt aus möglich ist, sondern eine Zusammenschau aus verschiedenen Standpunkten darstellt $t^{50}$. Man wird also die hier dargelegten Grundzüge der Art und Weise, wie Dürer Landschaften dargestellt hat, für die Betrachtung der Zeichnung des Aspergs voraussetzen müssen.

Dürers Zeichnung gibt uns wohl die älteste Darstellung des Aspergs, die Anspruch auf Wirklichkeitstreue machen kann, da sie viele Einzelheiten der Burg und des Städtleins bietet. Auf dem Berg befanden sich damals im Westen das Städtchen Asperg, gekennzeichnet durch die Kirche, im Osten die Burg, die von einem dicken runden Turm bestimmt wird. Da die Kirche sicher geostet war, handelt es sich um eine Chorturmkirche, bei der das Turmuntergeschoss den Chor bildete. Das Kirchenschiff ist durch einen niedrigeren Zwischenbau mit dem Turm verbunden. Westlich der Kirche, wo sich das Gelände absenkt, stehen einige kleinere Häuser, die teilweise durch die Beschießung beschädigt sind. Die Südwestecke der Mauer, die Burg und Stadt umfasst, scheint mit einem runden Turm befestigt gewesen zu sein.

Die wohl durch einen Graben markierte Grenze zwischen Städtchen und Burg lässt sich nicht genau erkennen. Möglicherweise wird diese Grenze durch den deutlich erkennbaren Rücksprung in der Umfassungsmauer, rechts von der großen Bresche, bezeichnet. Die Burg hat einen großen runden Bergfried, wie man ihn auch in Besigheim oder auf dem Reichenberg im Murrtal findet. An den Bergfried

${ }^{47}$ Vgl. das Kapitel „Bilderfindung und Naturvorgabe in Dürers Darstellung des „Tales bei Kalchreuth““”, bei Leber (wie Anm.46) S. 128-146.

${ }^{48}$ F.W. Hollstein, German Engravings, Etchings and Woodcuts ca. 1400-1700, Vol.7: Albrecht and Hans Dürer, hg. von K. G. Boond/R. W. Scheller, Amsterdam [1962], S. 66.

49 Leber (wie Anm. 46) S. 99 f., mit Abb.1-6.

50 Diese Annahme ist in neuerer Zeit wieder strittig. Während Hess (wie Anm. 44) S. 123, von zwei Standpunkten ausgeht, nimmt Grossmann, Die Architektur (wie Anm.44) S.226f. an, dass die Ansicht von einem einzigen Standpunkt aus gezeichnet worden sei. 
schließt sich östlich ein großes Gebäude an, das wohl als Palas der Burg angesprochen werden kann. Leider lässt sich diese Darstellung von Burg und Städtchen nicht mehr anderweitig belegen; das Städtchen wurde ja 1535 an den Fuß des Berges verlegt und der Berg durch den Festungsbau vollständig verändert.

Die Weinberge am Südhang des Aspergs mit ihren Terrassenmauern sind nur schematisch dargestellt. Dies stellt einen deutlichen Gegensatz zu der sorgfältigen Wiedergabe der Bebauung auf dem Berg dar. Am Fuß des Aspergs ist eine kleine Ansammlung von Häusern mit einem Ziehbrunnen zu erkennen, die mit Dürers Beischreibung als Tyfenpach bezeichnet wird. Dieses Tiefenbach hat man schon mit einem abgegangenen Hof identifiziert, auf den der Möglinger Flurname Dieppach hindeuten könnte ${ }^{51}$. Dies ist durchaus möglich, doch könnte hier auch eine Namensverwechslung vorliegen.

Den Vordergrund des Bildes nimmt links eine weitere Häusergruppe mit Kirche ein, bei der sich nach zwei Aufschriften der knecht leger befindet. Diese Andeutung eines Kirchdorfs wurde zumeist für Möglingen gehalten ${ }^{52}$. Dies ist nach der bereits beschriebenen Methode Dürers, Ansichten aus verschiedenen Blickpunkten zusammenzustellen, sicher zutreffend. Dafür spricht auch, dass dieser Vordergrund vom Mittelgrund durch eine sich durch die ganze Zeichnung ziehende Hangkante abgesetzt ist. Diese Kante ist einesteils durch Schraffuren kenntlich gemacht, andernteils ist sie durch die nur mit ihrer Giebelspitze sichtbaren Häuser links und die nur mit ihren Mündungen sichtbaren Kanonenrohre der linken Batterie zu erahnen. Es dürfte also die Auffassung, dass das Dorf Weichenberg mit der knecht leger gemeint ist, abzulehnen sein. Diese deutliche Unterteilung der Darstellung mit Möglingen im Vordergrund und den drei Batterien am Fuß des Berges stellt natürlich eine erhebliche Verkürzung der Tiefe des Raumes dar, die aber für Dürer nicht ungewöhnlich ist. Diese Verkürzung wird leicht übersehen, weil in den Proportionen keinerlei Rücksicht darauf genommen wird, da die Personen im Mittelgrund nur unwesentlich kleiner dargestellt sind, als die im Vordergrund.

Dieselbe erhebliche Verkürzung zeigt sich auch beim Hintergrund, der rechts vom Asperg nur durch eine Horizontlinie angedeutet ist, während links zwei Städte zu sehen sind, die als Gruningen (Markgröningen) und Pyeteka (Bietigheim) bezeichnet sind. Diese beiden Städte sind freilich nur symbolhaft dargestellt, sie zeigen nur die Kirchen, die von wenigen Häusern und einem von Türmen und Toren besetzten Mauerring umgeben sind. So ist aber die Landschaft von keinem Punkt südlich des Aspergs zu sehen, da der sich vom Asperg westlich in Richtung Markgröningen ziehende Höhenrücken weggelassen ist. Es dürfte damit klar sein, dass die Frage, von welchem Standort aus Dürer seine Zeichnung aufgenommen hat, müßig ist.

51 Kretzschmar (wie Anm. 8) S. 83, nach Römer, Markgröningen (wie Anm. 8) S. 236.

52 So schon Römer, Markgröningen (wie Anm.8) S.235; Burkhardt (wie Anm.8) S. 130 . 
Nun handelt es sich bei der Zeichnung des Aspergs aber nicht um eine Landschaft, wie die oben erwähnte Ansicht von Arco oder das Tal bei Kalchreuth, sondern um die Darstellung eines kriegerischen Geschehens, das sich in einem bestimmten Raum abspielt. Neben der knecht leger, womit wohl Möglingen gemeint ist, sehen wir drei unterschiedlich stark besetzte Batterien oder Schanzen, die durch mit Erde gefüllte Schanzkörbe gedeckt sind. Aus der gedruckten Beschreibung von 1519 wissen wir nicht nur, dass das Fußvolk des Bündischen Heeres in Möglingen, Pflugfelden und Tamm einquartiert war, sondern dass die Belagerer nacheinander drei Schanzen für die Artillerie errichteten. Die erste Schanze wurde am 16. Mai errichtet, die zweite, näher am Asperg, am 17. Mai, die dritte by Wychenberg am 21. Mai. Man wird wohl nicht fehlgehen, wenn man diese Reihenfolge auf dem Dürerschen Blatt von rechts nach links dargestellt sieht. Damit wäre die Annahme, dass mit dem Dürerschen Tyfenbach die Siedlung Weichenberg gemeint ist, am sinnvollsten in Einklang zu bringen.

Betrachtet man die Artilleriestellungen genauer, so fällt zunächst die nicht sehr zahlreiche Geschützbedienung auf; der Künstler ist wohl wegen der Skizzenhaftigkeit dieses Teils seiner Arbeit sparsam damit umgegangen. Auffälliger ist, dass viele Kanonen auf zwei Achsen stehen und auch feuern. Üblich waren die beiden Achsen nur für den Transport, es scheint aber auch möglich gewesen zu sein, in diesem Zustand zu schießen. Das ist wohl gemeint, wenn es in dem gedruckten Bericht zum 28. März von der Beschießung Heidenheims heißt ${ }^{53}$, man habe so bald das geschitz yns leger ist kom [m]en on geschantz [ohne zu schanzen] auff der Axt [Achse] zum schloß abgeschossen. Eine der Kanonen, die größte in der linken Batterie, hat überhaupt keine Räder, sondern ist offensichtlich fest eingebaut, wobei hinten ein Holzgestell zu bemerken ist, das den Rückstoß auffangen soll. Ganz offensichtlich handelt es sich um die grosse wirttenbergische pich $\beta$ genant der Bruder, die am 19. Mai eintraf und die man die nacht in die unttern schantz gelägert hat ${ }^{54}$.

Es ist damit eine bemerkenswerte Übereinstimmung dieses Berichts mit der bildlichen Darstellung Dürers festzustellen. Ebenso verhält es sich mit dem Geschehen auf der linken Seite der Zeichnung, am südwestlichen Abhang des Asperg. Man sieht hier zwei Mörser, die auf Gestellen montiert sind, und drei Kanonen in Aktion. Der eine der Mörser wird ausgeputzt, der andere gerade abgeschossen, ebenso wie eine der Kanonen. Als Ergebnis der Beschießung von dieser Seite gibt der 1519 gedruckte Bericht an, es sei ym Stättlin Asperg ain hauß vom schiessen eingefallen / hat 10 menschen erschlagen und ersteckt dan etlich pulver ist ym selben hau $\beta$ gelegen ist vom schiessen angezindt, hat den ym Stätlin grossen schrecken bracht $t^{55}$. Diese Stelle fehlt bei Stumphart, doch ist auf der Dürerschen Zeichnung das

${ }^{53}$ Hörzug (wie Anm. 17), Bl. A2 r, vgl. Sattlek (wie Anm.13) S. 31.

${ }^{54}$ Hörzug (wie Anm. 17), Bl.B3v-B4 r, vgl. SATtlen (wie Anm. 13) S. 40.

${ }^{55}$ Hörzug (wie Anm. 17), Bl.B4 r. 
Albrecht Dürer: Die Beschießung des Hohenaspergs 1519. Federzeichnung (Staatliche Museen zu Berlin, Kupferstichkabinett KdZ, 31).

[Die Abbildung kann aus rechtlichen Gründen online nicht bereitgestellt werden.]

Zeitschrift für Württembergische Landesgeschichte 75 (2016), S. 51-67

(C) Kommission für geschichtliche Landeskunde in Baden-Württemberg und Württembergischer Geschichts- und Altertumsverein e.V. 
zerstörte Haus ganz links, oberhalb des runden Turms gut zu erkennen. Die Beschießung wurde am folgenden Tag, dem 21. Mai noch verstärkt, an dem man als [Druckfehler für: aus] allen schantzen vast [heftig] geschossen und hat die 3 mörser oder poler ${ }^{56}$ gar an Asperg in holen weg gelägert und den tag zehen mal darauß mit stain und feürkugeln geworffen ${ }^{57}$. Auch die Situation des Hohlwegs ist auf der Dürerschen Zeichnung durch die Kanone angedeutet, von der man nur das Rohr und einen Teil der Räder oberhalb der Achse sieht.

Schließlich ist auch die Bresche in der Mauer von Dürer dargestellt worden, von der der Bericht zum 23. Mai als der lucken [...] an der mawr redet, die so weit war, daß pey 25 knecht hetten neben ainander möchten hinein lauffen, wo die graben ynnen und aussen und die böche nit vorbindert hette ${ }^{58}$. Es muss freilich dahingestellt bleiben, ob diese Lücke in der Mauer tatsächlich an der Südseite entstand oder nicht doch an der Mauer, die das Städtlein von der Burg trennte und somit hier nicht sichtbar ist.

Die Übereinstimmung des Berichts mit der Zeichnung von Dürer geht so weit, dass in der Umgebung des Aspergs nur die Orte dargestellt werden, die für das Geschehen von Bedeutung waren. Möglingen, das zugleich die Stelle von Pflugfelden und Tamm vertritt, als Lager des Fußvolks des Bündischen Heers haben wir schon genannt, ebenso Weichenberg als den Ort, bei dem die dritte Schanze aufgeworfen wurde. Somit erklärt sich auch, weshalb Bietigheim und Markgröningen auf der Zeichnung dargestellt werden. In Bietigheim war Herzog Wilhelm von Bayern zuerst im Quartier, um dann am 17. Mai ins Schloss nach Markgröningen umzuziehen.

Diese Beobachtungen geben nun doch einige Hinweise auf die Entstehung des Blattes. Gewiss hat sich Dürer für das Befestigungswesen interessiert, wie seine Schrift „Etliche underricht zu befestigung der stett schloß und flecken “59 von 1527 belegt. Hier zeigt er eine gewaltige Festung, die von einem ebenso gewaltigen Heer belagert wird ${ }^{60}$. Überdies enthält diese Schrift ein sehr genaues Bild von einer $\mathrm{Ka}$ none, außerdem muss an die einigermaßen rätselhafte Eisenradierung „Die große Kanone"61 erinnert werden, so dass man Dürer als Sachverständigen in diesen Angelegenheiten bezeichnen kann ${ }^{62}$. Gleichwohl dürfte Dürer diese Zeichnung nicht allein um seines Interesses willen angefertigt haben. Auch Dürers Kunst musste

\footnotetext{
56 Mörser oder Böller.

57 Hörzug (wie Anm. 17), Bl.B4 r, vgl. Sattler (wie Anm.13) S. 40.

58 Hörzug (wie Anm. 17), Bl. C1 r, vgl. Sattler (wie Anm.13) S. 41.

${ }^{59}$ Hollstein (wie Anm. 48) S.258, wo auch die verschiedenen Ausgaben dieses Werks verzeichnet sind.

60 Ebd., S. 220.

61 Ebd., S. 87. Vgl. dazu auch Adriani/Schmauder (wie Anm.4) S. 318 f., mit Abb.

${ }^{62}$ Die vor längerer Zeit vorgenommene Zuschreibung etlicher Skizzen südwestdeutscher Burgen, wie Kaltental bei Stuttgart und solcher im Elsass, die Max BACH, Albrecht Dürer in Württemberg, in: WVjH 10 (1887) S. 111 f. zur Annahme einer Reise Dürers durch Schwaben im Jahre 1515 veranlasst hat, ist hinfällig, seit diese Skizzen Hans Baldung Grien zugewiesen
} 
nach Brot gehen. Das wird deutlich in dem oben zitierten Brief Beheims an Pirckheimer, in dem Dürers Reisepläne beurteilt werden. Ein Argument gegen eine Reise nach England oder Spanien war, dass er keine Söhne habe und sich deshalb mit seinem bisher erworbenen Vermögen begnügen könne. Im Umkehrschluss heißt das, dass Dürer mit diesen Reisen Erwerbsabsichten verfolgte, wie er es ja mit der Niederländischen Reise auch tat. Dies war umso nötiger, da sein Mäzen Kaiser Maximilian verstorben war.

Man wird annehmen können, dass Dürer das Blatt an Ort und Stelle gezeichnet hat ${ }^{63}$. Dafür spricht die Genauigkeit, mit der Burg und Städtlein auf dem Berg wiedergegeben sind. Ein Vergleich mit den phantasievollen, hoch aufgetürmten Burgen, die den Hintergrund für zahlreiche seiner Arbeiten bilden, ergibt, dass die Darstellung von Burg und Stadt Asperg ausgesprochen wirklichkeitsnah ist. Die Annahme, dass Dürer Skizzen, die er sich hier gemacht hat, später auf diesem Blatt zusammengestellt hat, ist daher abzulehnen. Das Blatt selber hat schon den Charakter einer Skizze. Das Wesentliche ist jedoch detailgetreu dargestellt, alles Übrige verrät bei aller Skizzenhaftigkeit immer noch die sichere Hand des Meisters.

Da wir nun als bestimmt annehmen müssen, dass Dürer die Beschießung nicht selbst miterlebt hat und somit nicht als Kriegsberichterstatter angesprochen werden kann, war er auf Berichte von Augenzeugen angewiesen. Ein solcher Augenzeuge war ganz offensichtlich der Verfasser des 1519 gedruckten und von Stumphart verarbeiteten Textes. Die Übereinstimmungen haben gezeigt, dass Dürer diesen Text kannte, dass das Dürersche Blatt in unmittelbarer Beziehung zu dem Bericht des ungenannt gebliebenen Feldzugsteilnehmers steht.

Die neuere Forschung hat gezeigt, dass Dürers Werke in größeren Zusammenhängen gesehen werden müssen, so wie die Landschaftsmalerei des frühen Dürer literarische Bezüge aufweist ${ }^{64}$. Es ist deshalb sicher berechtigt, das hier besprochene Blatt nicht einfach als Reiseerinnerung zu sehen, sondern in Zusammenhänge zu stellen, die wir vorerst allerdings nur vermuten können. Möglich wäre, dass der 1519 in Augsburg gedruckte Bericht über den Feldzug mit einem Stich nach der Dürerschen Zeichnung illustriert werden sollte. Vielleicht war Herzog Wilhelm von Bayern als Mäzen zur Finanzierung des Drucks ausersehen, möglicherweise aber auch Georg von Frundsberg. Einer der beiden Herren könnte der Reiter mit federgeschmückter Kopfbedeckung auf dem mit Schabracke und Federbusch ausgestatteten Pferd sein, der unterhalb des hohlen Wegs nahe der linken Bildkante erscheint. Offenbar hatte aber weder Herzog Wilhelm noch Georg von

worden sind. Vgl. dazu Hans Klaiber, Dürer in Schwaben, in: Schwäbischer Merkur 302 vom 30. Juni/1. Juli 1928.

${ }^{63}$ Dieser Auffassung sind auch die Verfasser von Albrecht Dürer. Kritischer Katalog (wie Anm.1) S. 95.

64 Jörg Robert, Dürer, Celtis und die Geburt der Landschaftsmalerei aus dem Geist der »Germania illustrata«, in: Der frühe Dürer (wie Anm.44) S.65-77. 
Frundsberg das Bedürfnis, sich wie der verstorbene Kaiser Maximilian im „Weißkunig“ oder „Theuerdank“ und den anderen Publikationen, an denen Dürer mitgearbeitet hat ${ }^{65}$, mit seinen Taten im Druck verewigen zu lassen, so dass es zu einer solchen Veröffentlichung nicht gekommen ist ${ }^{66}$. So blieb es bei dem einigermaßen bescheidenen Augsburger Druck von 1519, dessen einziger Schmuck die aus vier Druckstöcken zusammengesetzte Titelrahmung ist. Aufwändiger ist die zeitgenössische Dokumentation des Feldzugs des Schwäbischen Bundes von 1523, mit der die Zerstörung einer Anzahl von Burgen in Franken im Bild vorgeführt wird ${ }^{67}$. Allerdings halten die einigermaßen groben Holzschnitte dieser Veröffentlichung einem Vergleich mit der Dürerschen Zeichnung nicht stand. Diese Zeichnung, die tatsächlich einmalig in Dürers Werk dasteht und sich als Illustration zu dem 1519 im Druck erschienenen Feldzugsbericht erwiesen hat, ist zweifellos die wichtigste frühe Darstellung des Aspergs vor seinem Ausbau zur frühneuzeitlichen Festung.

${ }^{65}$ Vgl. Götz Adriani, Kaiser Maximilian I. und die Bildmedien, in: Adriani/Schmauder (wie Anm.4) S.217-265.

${ }^{66}$ Immerhin gibt es im Hauptstaatsarchiv München eine Handschrift von Augustin Köllner, Beschreibung des Feldzugs Herzog Wilhelms von Baiern, gegen Herzog Ulrich im Herbst 1519; vgl. Wilhelm HeYd, Bibliographie der Württembergischen Geschichte, Bd.1, Stuttgart 1895, Nr.789, S. 84, die somit den hier in Rede stehenden Frühjahrsfeldzug nicht betrifft.

67 Vgl. dazu Thomas Steinmetz, Conterfei etlicher Kriegshandlungen von 1523 bis in das 1527 Jar. Zu Burgendarstellungen über die „Absberger Fehde“ oder den „Fränkischen Krieg“, in: Beiträge zur Erforschung des Odenwaldes und seiner Randlandschaften 4 (1986) S. $365-386$. 